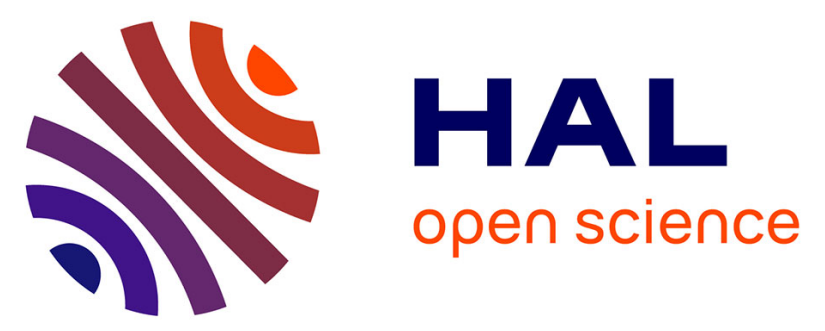

\title{
Dosage d'une couche adsorbée sur une pointe à émission de champ à l'aide de méthodes de microanalyse nucléaire ( $\mathrm{Pd}$ ou $\mathrm{C}$ adsorbé sur $\mathrm{W}$ )
}

\author{
A. Piquet, Hélène Roux, G. Pralong, J.P. Dupin
}

\section{- To cite this version:}

A. Piquet, Hélène Roux, G. Pralong, J.P. Dupin. Dosage d'une couche adsorbée sur une pointe à émission de champ à l'aide de méthodes de microanalyse nucléaire ( $\mathrm{Pd}$ ou $\mathrm{C}$ adsorbé sur $\mathrm{W})$. Revue de Physique Appliquée, 1980, 15 (1), pp.67-73. 10.1051/rphysap:0198000150106700 . jpa-00244700

HAL Id: jpa-00244700

https://hal.science/jpa-00244700

Submitted on 1 Jan 1980

HAL is a multi-disciplinary open access archive for the deposit and dissemination of scientific research documents, whether they are published or not. The documents may come from teaching and research institutions in France or abroad, or from public or private research centers.
L'archive ouverte pluridisciplinaire HAL, est destinée au dépôt et à la diffusion de documents scientifiques de niveau recherche, publiés ou non, émanant des établissements d'enseignement et de recherche français ou étrangers, des laboratoires publics ou privés. 


\title{
Dosage d'une couche adsorbée sur une pointe à émission de champ à l'aide de méthodes de microanalyse nucléaire (Pd ou $\mathrm{C}$ adsorbé sur $\mathrm{W}$ )
}

\author{
A. Piquet, H. Roux, G. Pralong et J. P. Dupin \\ Département de Physique des Matériaux $(*)$, Université Claude-Bernard, Lyon-I, \\ 43 bd du 11-Novembre-1918, 69621 Villeurbanne Cedex, France \\ (Reçu le 27 avril 1979, révisé le 28 septembre 1979, accepté le 2 octobre 1979)
}

\begin{abstract}
Résumé. - La micro-analyse nucléaire est utilisée pour déterminer la concentration moyenne d'une couche adsorbée sur une pointe à émission de champ. Le flux d'adsorbat provenant de la source est condensé simultanément sur la pointe et sur une cible adjointe jusqu'à l'obtention d'une singularité en émission de champ ; la sensibilité des méthodes nucléaires est telle que le dépôt reçu dans ces conditions par la cible est suffisant pour être dosé avec précision. Ainsi, dans le cas d'une pointe de tungstène, la concentration moyenne de palladium correspondant à l'obtention du maximum de travail de sortie moyen a été évaluée à $(0,60 \pm 0,06) 10^{15}$ atomes $\mathrm{Pd} / \mathrm{cm}^{2}$, et la concentration moyenne de carbone correspondant à l'apparition des facettes $\{334\}$ a été évaluée à $(1,5 \pm 0,3) 10^{14}$ atomes $\mathrm{C} / \mathrm{cm}^{2}$.
\end{abstract}

\begin{abstract}
Average concentration of adsorbed layers on F.E.M. tips is determined by means of nuclear microanalysis. The adsorbate source flux is condensed simultaneously on the tip and on an adjoining target until a F.E.M. singularity is obtained. Nuclear methods being very sensitive, target deposit is sufficient to obtain an accurate determination of the concentration. In the case of a tungsten tip, the average palladium concentration corresponding to the maximum average work function is $(0.60 \pm 0.06) 10^{15} \mathrm{Pd}$ atoms $/ \mathrm{cm}^{2}$, and the average carbon concentration corresponding to the appearance of the $\{334\}$ facets is $(1.5 \pm 0.3) 10^{14} \mathrm{C}$ atoms $/ \mathrm{cm}^{2}$.
\end{abstract}

1. Introduction. - Les problèmes liés à l'adsorption occupent une place importante dans la physicochimie des surfaces. Les moyens d'investigation modernes d'étude des surfaces tels que A.E.S., L.E.E.D., S.I.M.S., E.S.C.A., F.E.M., etc..., sont sensibles à des concentrations superficielles d'adsorbat de l'ordre de $10^{13}$ atomes $/ \mathrm{cm}^{2}$. Cependant, l'utilisation de ces techniques se heurte souvent au problème de la détermination de la concentration absolue de la couche adsorbée. Différentes méthodes indirectes de dosage permettent une telle détermination, mais il existe souvent des divergences entre les résultats obtenus à l'aide de l'une ou l'autre de ces méthodes.

Les développements récents de la micro-analyse nucléaire ont fait de cette technique l'une des méthodes les plus sensibles de dosage absolu. Nous l'avons utilisée pour déterminer des concentrations caractéristiques de singularités en microscopie électronique à émission de champ : obtention d'un extrémum du travail de sortie moyen, ou transition du dia-

(*) Associé au C.N.R.S. gramme d'émission électronique de champ par exemple. Nous présentons ici les résultats relatifs à l'adsorption de palladium ou de carbone sur une pointe de tungstène.

2. Position du problème. - De nombreuses études d'adsorption par microscopie à émission électronique de champ sont effectuées essentiellement avec des concentrations superficielles moyennes allant de quelque $10^{13}$ à quelque $10^{15}$ atomes $/ \mathrm{cm}^{2}$. Le dosage absolu de telles concentrations ne peut pas être effectué directement sur la pointe en raison de ses très petites dimensions ; aussi utilise-t-on la plupart du temps des méthodes de dosage indirectes qui manquent souvent de précision en raison même du principe qu'elles utilisent. En général ces méthodes reposent sur la calibration du flux de la source d'adsorbat soit in situ par microbalance, quartz vibrant, jauge Bayard-Alpert, etc..., soit extra situ par dosage du dépôt sur une cible adjointe par interférométrie optique, palpeur mécanique, absorption optique, etc... La plupart de ces méthodes exigent le cumul d'un grand nombre de doses d'adsorbat, une dose corres- 
pondant à la concentration nécessaire à l'obtention de la singularité en émission électronique de champ. L'utilisation de telles méthodes requiert soit une très bonne stabilité du flux de la source d'adsorbat, soit la connaissance des densités des dépôts en couche mince, soit les deux simultanément, ce qui peut entraîner une incertitude importante sur la concentration absolue correspondant à la dose.

La méthode de dosage du flux de la source par micro-analyse nucléaire ne présente pas les inconvénients des méthodes rappelées ci-dessus : elle permet de déterminer de façon absolue des concentrations superficielles de l'ordre de $10^{15}$ atomes $/ \mathrm{cm}^{2}$ avec une précision pouvant atteindre $5 \%$ dans les cas favorables.

3. Rappels sur le principe de dosage par microanalyse nucléaire. - Deux types d'analyse sont aujourd'hui très utilisés pour l'identification et le dosage d'éléments localisés sur ou au voisinage de la surface d'une cible de nature différente; ce sont respectivement :

- l'analyse par rétrodiffusion d'ions légers (diffusion élastique) [1],

- l'analyse par observation directe d'une réaction nucléaire $[1,2,3]$.

Elles consistent toutes deux en l'analyse du résultat de l'interaction d'un faisceau de particules primaires avec l'élément de la cible à doser. Dans le premier type, l'information est portée par les particules ayant rebondi sur la cible, par contre dans le deuxième type, elle est portée par les particules secondaires résultant d'une réaction nucléaire spécifique de l'élément à caractériser. Dans la pratique le type d'analyse utilisé est choisi en fonction de la nature de l'élément à doser. Les ions du faisceau primaire, accélérés par un accélérateur de type Van de Graaff, ont une énergie de l'ordre du $\mathrm{MeV}$; un ensemble détecteursélecteur multicanaux permet d'analyser en énergie les particules issues de la cible dans une certaine direction (Fig. 1).

3.1 L'ANALYSE PAR RÉTRODIFFUSION D'IONS LÉGERS. - Un ion de masse $M$ (de numéro atomique $Z$ ) bombardant perpendiculairement une cible avec une énergie $E_{0}$ est rétrodiffusé élastiquement par celle-ci

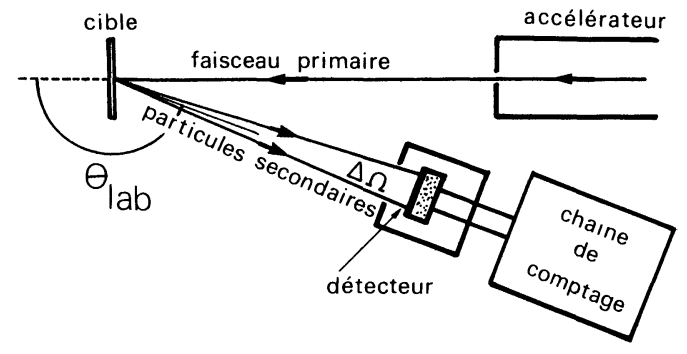

Fig. 1. - Schéma de la chambre d'analyse.

[The schematic draw of nuclear analysis chamber device.] dans une direction faisant un angle $\theta$ avec sa direction incidente avec une énergie $E$ telle que :

$$
E=k^{2} E_{0}
$$

avec $k=f\left(M, M^{\prime}, E_{0}, \theta\right)$.

Dans l'hypothèse simplificatrice où $\theta$ est voisin de $180^{\circ}$

$$
k=\frac{M^{\prime}-M}{M^{\prime}+M}
$$

où $M^{\prime}$ est la masse de l'atome diffusant, de numéro atomique $Z^{\prime}$. Lorsque les conditions expérimentales $M, E_{0}, \theta$ sont fixées, $E$ est uniquement une fonction de $M^{\prime}$.

Considérons le cas d'une cible plane constituée d'une matrice $m$ contenant sur ou au voisinage de sa surface (quelques centaines d'angströms par exemple) un élément i (impureté à doser) plus lourd que celui de la matrice. On conçoit que le spectre énergétique des ions rétrodiffusés, dont un schéma est donné à la figure 2, permet de séparer en masse les deux éléments. $\mathrm{Si}$, en outre, on tient compte de la probabilité (section efficace de diffusion) pour qu'un ion soit rétrodiffusé par l'un ou l'autre des deux éléments et de la perte en énergie (pouvoir d'arrêt) qu'a subi l'ion avant d'être diffusé par un atome, l'analyse en énergie des ions rétrodiffusés va nous renseigner sur le nombre d'atomes diffusants et leur localisation en profondeur.

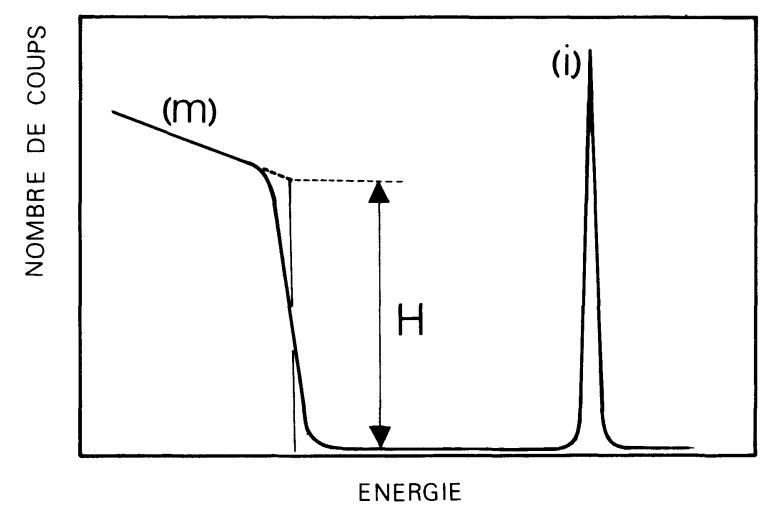

Fig. 2. - Allure du spectre en énergie des ions rétrodiffusés par une cible comportant une très mince couche d'impureté localisée sur sa surface.

[Characteristics of backscattering ions energy spectrum in the case of a target having a thin impurity layer at the surface.]

Les ions rétrodiffusés dans une tranche d'épaisseur $\mathrm{d} x$ à une profondeur $x$ de la cible sont détectés dans un angle solide $\Delta \Omega$ dans une direction moyenne $\theta$ par un détecteur et une chaîne de comptage et se traduisent dans le spectre en énergie par un nombre de coups $\mathrm{d} n(E)$ correspondant à une énergie comprise entre $E$ et $E+\mathrm{d} E$ selon la relation :

$$
\mathrm{d} n(E)=\eta N(x)\left(\frac{\mathrm{d} \sigma}{\mathrm{d} \Omega}\right)_{E} \Delta \Omega \mathrm{d} x
$$


dans laquelle :

- $\eta$ est le nombre d'ions incidents,

- $N(x)$ est la concentration de l'élément diffusant à la profondeur $x$,

- $(\mathrm{d} \sigma / \mathrm{d} \Omega)_{E}$ la section efficace de rétrodiffusion pour l'énergie $E$ qui dans le cas d'une interaction purement coulombienne est donnée par la formule de Rutherford

$$
\begin{aligned}
\left(\frac{\mathrm{d} \sigma}{\mathrm{d} \Omega}\right)_{E}=1,3 & \times 10^{-27}\left(\frac{Z Z^{\prime}}{E}\right)^{2} \times \\
& \times\left(\frac{M+M^{\prime}}{M^{\prime}}\right)^{2} \frac{1}{\sin ^{2} \frac{\theta}{2}} \mathrm{~cm}^{2} / \mathrm{Str} .
\end{aligned}
$$

Dans le cas particulier d'une couche très mince d'impureté (de l'ordre d'une centaine d'angströms) localisée à la surface de la matrice et caractérisée par le pic (i) de la figure $2, \mathrm{~d} \sigma / \mathrm{d} \Omega$ est une constante, l'intégration de (3) donne immédiatement :

$$
\int_{E} \mathrm{~d} n(E)=\eta \frac{\mathrm{d} \sigma}{\mathrm{d} \Omega} \Delta \Omega \int_{x} N(x) \mathrm{d} x
$$

soit

$$
A=\eta\left(\frac{\mathrm{d} \sigma}{\mathrm{d} \Omega}\right)_{\mathrm{i}} \Delta \Omega N_{\mathrm{i}}
$$

où $A$ est l'aire sous le pic (i) (en nombre de coups) et $N_{\mathrm{i}}$ est le nombre d'atomes $/ \mathrm{cm}^{2}$.

Dans le cas de la matrice épaisse $m$, caractérisée sur la figure 2 par un front de montée de hauteur $H$ (en nombre de coups/canal) et un plateau, l'expression (3) donne directement

$$
H=\eta\left(\frac{\mathrm{d} \sigma}{\mathrm{d} \Omega}\right)_{\mathrm{m}} \Delta \Omega N_{\mathrm{m}} \delta x
$$

où $N_{\mathrm{m}}$ est la concentration des atomes de la matrice et $\delta x$ est l'épaisseur de la matrice correspondant à un canal c'est-à-dire à un intervalle d'énergie $\delta E$.

La connaissance du pouvoir d'arrêt de la matrice

$$
S_{\mathrm{m}}=\left(\frac{\mathrm{d} E}{\mathrm{~d} x}\right)_{\mathrm{m}}
$$

permet d'écrire

$$
\delta x=\frac{\delta E}{\left(\frac{\mathrm{d} E}{\mathrm{~d} x}\right)_{\mathrm{m}}}
$$

d'où

$$
H=\eta\left(\frac{\mathrm{d} \sigma}{\mathrm{d} \Omega}\right)_{\mathrm{m}} \Delta \Omega N_{\mathrm{m}} \frac{\delta E}{S_{\mathrm{m}}} .
$$

La combinaison de (6) et de (10) donne :

$$
N_{\mathrm{i}}=N_{\mathrm{m}} \frac{A}{H} \frac{\left(\frac{\mathrm{d} \sigma}{\mathrm{d} \Omega}\right)_{\mathrm{m}}}{\left(\frac{\mathrm{d} \sigma}{\mathrm{d} \Omega}\right)_{\mathrm{i}}} \times \frac{\delta E}{S_{\mathrm{m}}} .
$$

On voit que le spectre d'analyse en énergie des ions rétrodiffusés permet de déterminer directement et de façon absolue la concentration d'atomes d'impuretés à la surface d'une matrice dont la nature, la masse volumique et le pouvoir d'arrêt sont connues.

Dans le cas où l'impureté est répartie sur une très faible profondeur, le pic (i) est un pic fin dont la largeur correspond pratiquement à la résolution en énergie de l'ensemble de détection des ions rétrodiffusés; si l'impureté est répartie sur une profondeur plus importante le pic est élargi du côté des basses énergies.

3.2 L'ANALYSE PAR OBSERVATION DIRECTE D'UNE RÉACTION NUCLÉAIRE. - Dans ce type d'analyse les particules primaires qui bombardent les noyaux des atomes de la cible provoquent des réactions nucléaires en donnant de nouveaux noyaux et des particules secondaires (ainsi qu'un rayonnement) dont la nature et l'énergie sont caractéristiques de l'élément bombardé. L'analyse en énergie de ces particules permet de séparer certains éléments constitutifs de la cible et de déterminer leur distribution en profondeur (sur environ $1 \mu \mathrm{m}$ ) [4]. En pratique l'interprétation de la partie du spectre énergétique correspondant à un élément se fait de manière analogue à celle de la rétrodiffusion à l'aide de la relation (3). La section efficace de la réaction $\mathrm{d} \sigma / \mathrm{d} \Omega$ est une fonction de l'énergie des particules incidentes qui présente souvent des paliers : en se plaçant au maximum d'énergie d'un de ces paliers et si l'étendue de ce dernier permet à la section efficace de rester constante sur toute l'épaisseur du dépôt à doser, l'équation (3) se réduit à :

$$
A=\eta \frac{\mathrm{d} \sigma}{\mathrm{d} \Omega} \Delta \Omega N_{\mathrm{i}}
$$

le dosage de l'impureté revient alors à la détermination de l'aire sous le pic caractéristique de l'élément.

En général le terme $\eta \frac{\mathrm{d} \sigma}{\mathrm{d} \Omega} \Delta \Omega$, qui dépend des conditions d'analyse et de détection, est éliminé en effectuant dans les mêmes conditions un dosage du même élément sur une cible étalon.

3.3 CHOIX DU TYPE D'ANALYSE. - En pratique, on utilise la rétrodiffusion d'ions légers $\left(\alpha,{ }^{7} \mathrm{Li},{ }^{12} \mathrm{C}\right.$, ${ }^{16} \mathrm{O}$ par exemple) pour doser un élément lourd sur une cible constituée d'un élément léger et l'analyse par observation directe d'une réaction nucléaire dans le cas du dosage d'un élément léger $\left(Z^{\prime}<30\right)$ sur une cible d'un élément lourd. La sensibilité peut atteindre $10^{11}$ atomes $/ \mathrm{cm}^{2}$ dans les cas favorables [4] pour le premier type d'analyse et $10^{13}$ atomes $/ \mathrm{cm}^{2}$ pour le deuxième type [2] ; il est donc relativement facile de doser des couches minces (de quelques $10^{15}$ atomes $/ \mathrm{cm}^{2}$ ) strictement localisées à la surface d'une cible.

La précision optimale des dosages que l'on peut effectuer avec de telles méthodes est de l'ordre de 
$5 \%$. Il est à noter que le rendement de réaction des deux types de méthode est indépendant de la liaison chimique des éléments à doser, ce qui constitue un avantage par rapport à d'autres méthodes (comme la spectroscopie Auger par exemple). En putre compte tenu des énergies des particules du faisceau primaire la perte de matière par pulvérisation à la surface est négligeable. L'élément de surface de la cible nécessaire à l'analyse est de l'ordre de $1 \mathrm{~mm}^{2}$.

4. Mode expérimental. - La pointe émettrice du microscope, obtenue par taille électrolytique d'un fil monocristallin de tungstène, a la forme d'un cône de révolution de demi-angle voisin de $4^{\circ}$ et de longueur de l'ordre de $1 \mathrm{~mm}$, le rayon de courbure à l'extrémité quasi hémisphérique est voisin de $5000 \AA$. Elle est portée par une boucle également en tungstène qui permet son chauffage par effet Joule.

La source d'adsorbat est disposée dans le microscope de telle manière que le flux d'atomes qu'elle émet soit perpendiculaire à l'axe de la pointe et à une cible plane destinée à recevoir le dépôt à doser (Fig. 3).
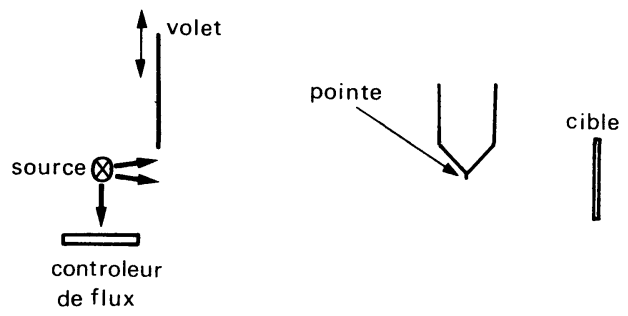

écran

Fig. 3. - Schéma du microscope à émission de champ.

[The schematic draw of field emission microscope device.]

Les distances cible-source $d_{1}$ et pointe-source $d_{2}$ sont assez grandes vis-à-vis des dimensions intrinsèques de la source pour que celle-ci puisse être considérée comme ponctuelle (une telle propriété est établie par la vérification avec une excellente précision de la loi en inverse carré de la distance en faisant varier la position de la source).

Avant le dépôt d'adsorbat la pointe est chauffée à haute température $(2600 \mathrm{~K})$ de manière à obtenir un profil d'équilibre thermique et une surface propre [6].

Après condensation d'adsorbat sur la partie de la pointe en vue optique de la source, un traitement thermique est effectué de manière à répartir le dépôt par diffusion de surface sur l'ensemble de la surface de la pointe. Un équilibre est obtenu après une durée de traitement thermique qui est fonction de la température de la pointe et de la nature de l'adsorbat. (quelques secondes à $800 \mathrm{~K}$ pour $\mathrm{Pd} / \mathrm{W}$ et à $1200 \mathrm{~K}$ pour $\mathrm{C} / \mathrm{W}$ ). L'obtention de cet équilibre et son maintien pendant plus d'une heure sont vérifiés par la constance (meilleure que $0,1 \%$ ) de la tension $V$ nécessaire à l'extraction, à la température ambiante, d'un courant total d'émission de champ donné. Ceci implique que la perte d'atomes d'adsorbat par désorption ou dissolution dans le volume de la pointe est négligeable. En effet, le courant d'émission de l'extrémité de la pointe dépendant exponentiellement du travail de sortie moyen $\left(\bar{\varphi}^{3 / 2}\right)$ et de la tension d'extraction $V$, toute variation sensible du premier paramètre - autrement dit de la concentration moyenne d'adsorbat - se traduirait par une variation corrélative du second.

La distribution de l'adsorbat à l'équilibre ne correspond pas à une concentration uniforme sur toute la surface de la pointe car celle-ci présente à son extrémité une grande variété de plans cristallographiques. Une singularité en émission de champ (extremum du travail de sortie moyen ou transition du diagramme d'émission de champ) est donc obtenue pour une certaine concentration superficielle moyenne d'adsorbat qui correspond à une distribution particulière de concentrations locales différentes. Cette concentration moyenne est un point de repère intéressant car il est reproductible d'une expérience à l'autre.

En conséquence, des dépôts successifs d'adsorbat sont effectués sur la pointe et répartis par diffusion de surface. La succession des dépôts est arrêtée lors de l'obtention de la singularité d'émission de champ. La cible est alors retirée du microscope et introduite pour dosage dans la chambre d'analyse de l'accélérateur.

En admettant l'égalité des coefficients de collage des adatomes sur la cible et sur la pointe (point qui sera discuté plus loin) et en tenant compte de la géométrie de révolution de la pointe et des distances $d_{1}$ et $d_{2}$, la concentration superficielle moyenne d'adsorbat $\bar{n}$ sur la surface de la pointe est telle que :

$$
\bar{n}=\frac{1}{\pi}\left(\frac{d_{1}}{d_{2}}\right)^{2} N_{\mathrm{i}}
$$

où $N_{\mathrm{i}}$ est la valeur de la concentration d'adsorbat obtenue par l'analyse de la cible.

5. Dosage du recouvrement moyen de palladium sur une pointe de tungstène. - La source de palladium est constituée d'un morceau de fil de pureté 99,999\% (Johnson Matthey, Specpur) inséré dans une hélice de tungstène chauffée par effet Joule [5]. Le flux de palladium évaporé, contrôlé par quartz vibrant, est envoyé simultanément sur la pointe et sur la cible constituée d'une feuille d'aluminium (pureté $99,99 \%$ ) dont une partie a été masquée pour servir de témoin. Le dépôt est effectué jusqu'à l'obtention de la valeur maximale $4,92 \mathrm{eV}$ du travail de sortie moyen de la pointe, valeur mesurée à la température ambiante, après uniformisation des dépôts à $800 \mathrm{~K}$. 
La cible est alors analysée par rétrodiffusion d'ions ${ }^{7} \mathrm{Li}^{+}$[7] d'énergie $2 \mathrm{MeV}$. La figure 4 montre le spectre d'analyse obtenu. On y distingue un pic, spécifique du palladium, qui est ici centré sur $1,54 \mathrm{MeV}$, un pic plus petit à $1,75 \mathrm{MeV}$ dénotant la présence de tungstène, et enfin un plateau débutant par un front de montée à $0,69 \mathrm{MeV}$, correspondant à l'aluminium de la cible. Ces deux pics, qui n'ont pas été observés sur la partie témoin de la cible, sont donc spécifiques de la couche déposée (les traces de tungstène détectées proviennent de l'hélice de la source). En raison de la très faible épaisseur de la couche de palladium à doser, strictement localisée à la surface de la cible, la perte en énergie des ions à la traversée de la couche est négligeable : la cible d'aluminium est analysée dans les mêmes conditions que le dépôt (en particulier, le terme $\eta \Delta \Omega$ est conservé à la traversée de la couche).

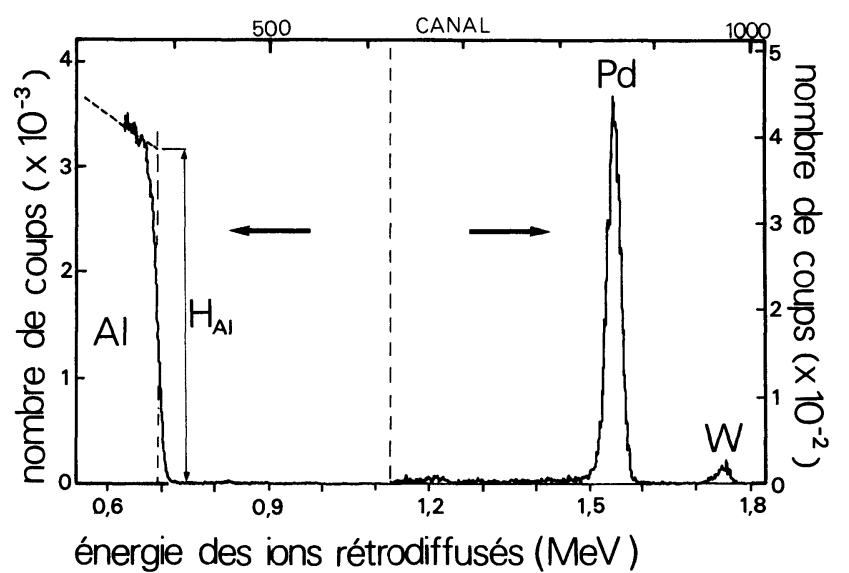

Fig. 4. - Spectre d'analyse en énergie des ions ${ }^{7} \mathrm{Li}^{+}$de $2 \mathrm{MeV}$ rétrodiffusés par une cible d'aluminium recouverte d'une couche adsorbée de palladium

(angle de détection $\theta_{1 \mathrm{lab}}=160^{\circ}$, résolution $=1,8 \mathrm{keV} /$ canal) .

Les flèches indiquent les échelles utilisées pour tracer le spectre.

[Backscattering energy spectrum of $2 \mathrm{MeV}^{7} \mathrm{Li}^{+}$ions for an $\mathrm{Al}$ target with adsorbed Pd

(detection angle $: \theta_{\text {lab }}=160^{\circ}$, resolution : $1.8 \mathrm{keV} / \mathrm{canal}$ ).

The arrows indicate the two different scales.]

Compte tenu des relations (11) et (13), le maximum de travail de sortie moyen est obtenu pour une concentration moyenne sur la pointe :

$$
\bar{n}_{\Phi_{\max }}=(0,60 \pm 0,06) 10^{15} \text { atomes } \mathrm{Pd} / \mathrm{cm}^{2} .
$$

Les traces de tungstène détectées simultanément correspondent à une concentration superficielle voisine de $2 \times 10^{13}$ atomes $\mathrm{W} / \mathrm{cm}^{2}$.

6. Dosage du carbone sur une pointe de tungstène. La source de carbone est constituée d'une spire de carbone chauffée par effet Joule. Le flux de carbone, contrôlé par un spectromètre de masse quadrupolaire, est évaporé simultanément sur la pointe et sur la cible constituée d'une plaquette de tungstène (de manière à avoir le même coefficient de collage sur la cible et sur la pointe). Au préalable, la surface de la cible a subi un traitement thermique à haute température sous ultravide $\left(10^{-11}\right.$ torr) puis à $1800 \mathrm{~K}$ en présence d'une pression partielle d'oxygène. Ce traitement a pour effet de nettoyer la surface et le volume de la cible par ségrégation, oxydation à la surface et désorption des impuretés (en particulier du carbone $[8,9])$. La pointe est portée à $1200 \mathrm{~K}$ pour répartir le dépôt sur l'ensemble de sa surface. L'observation, à la température ambiante, du diagramme d'émission de champ révèle l'existence $[10,11,12]$ de facettes $\{334\}$ à partir d'une concentration superficielle moyenne de carbone caractéristique et reproductible que nous avons adoptée comme dose unitaire.

La méthode choisie pour doser le carbone reçu par la cible consiste à bombarder celle-ci par des deutons, et à analyser les protons qui résultent de la réaction ${ }^{12} \mathrm{C}(\mathrm{d}, \mathrm{p}){ }^{13} \mathrm{C}$ [13]. L'énergie de $980 \mathrm{keV}$, choisie pour le faisceau primaire de deutons, correspond à un palier de section efficace de la réaction nucléaire. Les protons résultant de la réaction ont une énergie caractéristique voisine de $3 \mathrm{MeV}$.

Le détecteur à semi-conducteur est protégé des deutons rétrodiffusés par une feuille de mylar aluminisée d'épaisseur $25 \mu \mathrm{m}$ qui produit une perte en énergie de $0,5 \mathrm{MeV}$ des protons détectés.

Malheureusement, dans nos conditions expérimentales, une analyse préliminaire de la face de la cible qui n'a pas reçu le flux de la source révèle un apport de carbone supplémentaire par polymérisation des hydrocarbures de l'enceinte de l'accélérateur $\left(10^{-6}\right.$ torr) sous l'impact du faisceau de deutons. La comparaison des analyses effectuées sur les deux faces de la cible n'a pas permis de déterminer avec une précision satisfaisante la concentration correspondant à la dose. En conséquence, l'expérience a été reprise en déposant 30 doses sur la pointe. L'analyse nucléaire a été effectuée successivement sur les deux faces de la cible en suivant la cinétique de croissance $\mathrm{du}$ pic du carbone (un tel pic, centré vers $2,5 \mathrm{MeV}$, est présenté sur le spectre de la figure 5). La reproductibilité des comptages en changeant de point d'impact du faisceau de deutons sur chacune des deux faces de la cible est meilleure que $4 \%$. La linéarité et le parallélisme des deux cinétiques (Fig. 6) montre que l'apport de carbone au point d'impact du faisceau est proportionnel au nombre de deutons incidents. L'ordonnée à l'origine de la cinétique, relevée pour la face n'ayant pas reçu le dépôt à doser, pourrait correspondre à la présence du carbone résiduel dans la cible. Cependant compte tenu de la perte en énergie $(0,5 \mathrm{MeV})$ des protons dans la feuille de mylar, le pic de carbone détecté à $2,5 \mathrm{MeV}$ correspond en fait à des protons de $3 \mathrm{MeV}$ qui est l'énergie caracté- 


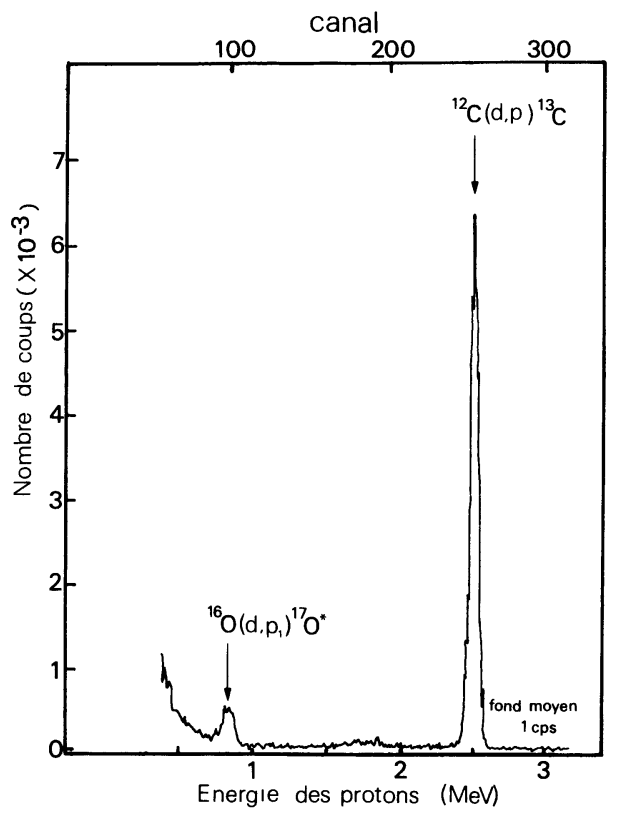

Fig. 5. - Spectre d'analyse en énergie des protons résultant du bombardement d'une cible de tungstène recouverte d'une couche adsorbée de carbone par des deutons de $980 \mathrm{keV}$ (angle de détection $\theta_{\text {lab }}=160^{\circ}$, résolution $=1,8 \mathrm{keV} /$ canal).

[Proton energy spectrum due to nuclear reaction of $980 \mathrm{keV}$ deuterons on a carbon adsorbed tungsten target (detection angle : $\theta_{\text {lab }}=160^{\circ}$, resolution : $1,8 \mathrm{keV} /$ canal).]

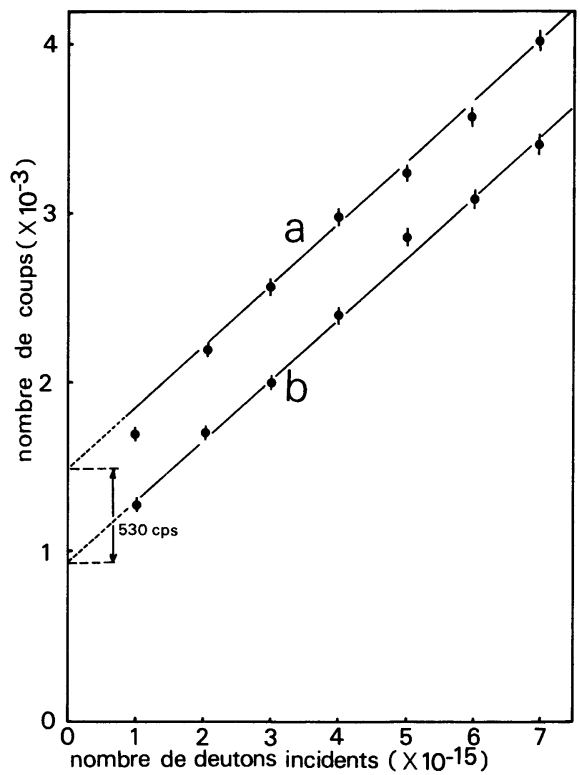

Fig. 6. - Cinétique de croissance du pic de carbone en fonction du nombre de deutons incidents obtenus : $a$ ) pour la face de la cible qui a reçu le dépôt à doser, $b$ ) pour la face opposée (les barres d'erreur correspondent à $2 \sqrt{v}$, $v$ étant le nombre de coups comptés).

[Carbon peak as a function of the incident deuterons flux $: a$ ) for the face which have received the carbon deposit, $b$ ) for the opposite face (the error bars correspond to $2 \sqrt{v}$, where $v$ is the number of cps).]

ristique de la réaction utilisée. Ceci indique que le carbone détecté est localisé à la surface (ou très près de celle-ci); il aurait alors pour origine la couche d'hydrocarbures condensée avant le début de l'analyse. En outre la forme acérée du pic, qui correspond à la résolution intrinsèque de la chaîne de détection, indique que le dépôt est très mince : une distribution sur une plus grande profondeur dans la cible du tungstène se traduirait par un élargissement de ce pic du côté des basses énergies. La différence des ordonnées des deux cinétiques de la figure 6 correspond alors au nombre de coups dus exclusivement à la couche de carbone déposée sous ultra-vide. Le dosage absolu de cette couche est obtenu à partir de la relation (12), dans laquelle le facteur $\eta \Delta \Omega \frac{\mathrm{d} \sigma}{\mathrm{d} \Omega}$ est éliminé en effectuant, dans les mêmes conditions d'analyse, un comptage sur une cible étalon. Celle-ci est constituée d'une couche homogène de carbone suffisamment épaisse pour que sa concentration superficielle soit déterminée par micropesée avec une précision voisine de $5 \%$.

Compte tenu des conditions géométriques (relation (13)) et du fait que 30 doses ont été déposées, la concentration moyenne $\bar{n}$ de carbone correspondant à l'apparition des facettes $\{334\}$ sur la pointe est :

$$
\bar{n}=(1,5 \pm 0,3) 10^{14} \text { atomes } \mathrm{C} / \mathrm{cm}^{2} \text {. }
$$

L'erreur de $20 \%$ a été estimée en attribuant $10 \%$ d'erreur au dosage sur la cible et à sa comparaison avec la cible étalon, $5 \%$ à la constitution de la cible étalon et $5 \%$ aux conditions géométriques d'évaporation dans le microscope à émission de champ.

Le spectre de la figure 5 présente en outre un petit pic à $0,8 \mathrm{MeV}$ dénotant la présence de traces d'oxygène résultant sans doute du nettoyage de la cible par traitement thermique sous oxygène.

7. Discussion. - Les méthodes nucléaires utilisées ici (comme les autres méthodes invoquées par ailleurs) pour doser une couche adsorbée sur une pointe à émission de champ ne sont pas des méthodes directes puisqu'elles nécessitent un dosage sur une cible intermédiaire. La précision du dosage est donc tributaire des conditions d'évaporation sur la pointe et sur la cible de nature différente et en particulier de l'égalité de leur coefficient de collage. Dans le cas du carbone sur une pointe de tungstène, cet inconvénient n'est pas à considérer car la cible utilisée est également en tungstène. En ce qui concerne le dépôt de palladium sur la pointe de tungstène et la cible d'aluminium, nous avons supposé que le coefficient de collage était le même et égal à l'unité. Cette hypothèse est généralement admise $[14,15]$ dans le cas de la condensation de métaux sur un substrat métallique à des températures très inférieures à la température de désorption de l'adsorbat. Le coefficient de condensation proprement dit, c'est-à-dire la proportion des atomes qui, ayant subi des collisions inélastiques avec la surface, restent piégés, a été 
déterminé expérimentalement et trouvé égal à 1 (à $1 \%$ près) dans le cas de l'argent sur le tungstène [15]. Le nombre d'atomes qui subissent des collisions élastiques peut être considéré comme négligeable par rapport au nombre de ceux qui subissent des collisions inélastiques en raison de la très forte énergie d'adsorption [15] : 4,5 eV/atome pour $\mathrm{Pd} / \mathrm{W}$ [5] et $7,4 \mathrm{eV} /$ atome pour $\mathrm{C} / \mathrm{W}$ [17] dans le cas de concentration de l'ordre de $10^{15}$ atomes $/ \mathrm{cm}^{2}$. En outre, en raison de la forte rugosité de la pointe qui présente environ $1 / 3$ de sites d'adsorption en décrochement [11], le coefficient de collage peut être considéré comme pratiquement constant quelle que soit l'incidence des atomes provenant de la source sur la pointe qui est quasi cylindrique.

En ce qui concerne les méthodes nucléaires proprement dites, mises à part les incertitudes liées à la détection et la statistique de comptage, la précision du dosage est, en fait, limitée uniquement par la précision sur la valeur du pouvoir d'arrêt de la cible $(\sim 5 \%)$ dans la rétrodiffusion d'ions (le rapport des sections efficaces de la relation (11) étant connu à $1 \%$ environ) et par la précision sur la concentration de la cible étalon dans le cas de la réaction nucléaire (l'imprécision sur la valeur de la section efficace n'intervenant pas du fait de son élimination). L'utilisation des méthodes nucléaires pour le dosage de couches très minces d'adsorption est donc intéressante en raison non seulement de leur sensibilité et de leur précision (les couches effectivement dosées sur les cibles étant respectivement de $2,54 \times 10^{15}$ atomes $\mathrm{Pd} / \mathrm{cm}^{2}$ et $4,5 \times 10^{15}$ atomes $\mathrm{C} / \mathrm{cm}^{2}$ ) mais également parce que ce sont des méthodes de dosage absolu qui permettent de déterminer directement la concentration d'atomes adsorbés sur les cibles.

Enfin, il convient de remarquer que la micro- analyse nucléaire peut être utilisée sans précaution particulière dans tous les cas où le transfert de la cible depuis l'enceinte du microscope à émission de champ jusqu'à la chambre d'analyse de l'accélérateur n'entraîne ni perte du dépôt, ni contamination superficielle par un composé chimique contenant l'élément à doser.

8. Conclusion. - Nous avons montré que la microanalyse nucléaire permet de déterminer avec une précision satisfaisante la concentration superficielle moyenne d'une couche adsorbée sur une pointe à émission de champ. Les dosages ont été effectués lorsque la concentration d'adsorbat correspond à l'obtention d'une singularité en émission de champ. Nous avons ainsi établi que le travail de sortie moyen d'une pointe de tungstène recouverte de palladium présente un maximum pour une concentration moyenne de $(0,60 \pm 0,06) 10^{15}$ atomes $\mathrm{Pd} / \mathrm{cm}^{2}$. De même, dans le cas d'une adsorption de carbone, l'observation du diagramme d'émission de champ révèle l'apparition des facettes $\{334\}$ lorsque la surface de la pointe de tungstène contient en moyenne $(1,5 \pm 0,3) 10^{14}$ atomes $\mathrm{C} / \mathrm{cm}^{2}$. Bien que l'obtention de ce dernier résultat ait nécessité quelques précautions particulières, la micro-analyse nucléaire s'est avérée être une technique particulièrement adaptée au dosage de concentrations superficielles de l'ordre de la monocouche.

Remerciements. - Nous remercions M. A. Cachard d'avoir bien voulu nous initier à la micro-analyse nucléaire. Les dosages ont été effectués à l'aide d'un accélérateur Van De Graaff de l'Institut de Physique Nucléaire de l'Université Lyon I.

\section{Bibliographie}

[1] Tousset, J., II ${ }^{e}$ Colloque International sur les applications des sciences et techniques du vide aux revêtements et états de surface. Congrès Avires II, Lyon (1972), supplément à $L E V I D E \mathrm{n}^{\circ} 158$.

Cachard, A., Thomas, J. P., in Nato Advanced Study Institute Series, Series B Physics 28 (1976) 367 ; Material characterization using ions beams. Edited by J. P. Thomas and A. Cachard (Plenum Press London-New York)

[2] Amsel, G., Nadai, J. P., D'Artemare, E., David, D., Girard, E., Moulin, J., Nucl. Instrum. Methods 92 (1971) 481.

[3] Amsel, G., J. Radioanal. Chem. 17 (1973) 15.

[4] Abel, F., Amsel, G., Bruneaux, M., Cohen, C., Maurel, A., Rigo, S., Roussel, J., J. Radioanal Chem. 16 (1973) 567.

[5] Roux, H., Piquet, A., Pralong, G., Uzan, R. et Drechsler, M., Surf. Sci. 71 (1978) 375.

[6] Piquet, A., Roux, H., Vu Thien Binh, Uzan, R. et DRECHSLER, M., Surf. Sci. 44 (1974) 575.

[7] Thomas, J. P., CaChard, A., Fallavier, M., TARdy, J., Marsaud, S. et Pivot, J., Revue Phys. Appl. 11 (1976) 65.

[8] Becker, J. A., Becker, J. et Brands, R. G., J. Appl. Phys. 32 (1961) 3
[9] Joyner, R. W., Rickman, J. et Roberts, M. W., Surf. Sci. 39 (1973) 445.

[10] MÜLlER, E. W., Ergeb. Exakten Naturwiss. Bd XXVII (1953) 1290.

[11] Bettler, P. C., Bennum, D. H. et Case, C. M., Surf. Sci. 44 (1974) 360 .

[12] Piquet, A., Pralong, G., Roux, H., Uzan, R. et Drechsler,

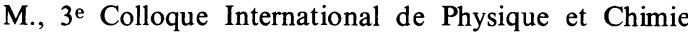
des Surfaces Solides. Suppl. au no 185 de $L E$ VIDE, Les Couches Minces p. 376.

[13] Beranger, G., David, D., Garcia, E. A. et Lucas, X. Revue Phys. Appl. 70 (1975) 87.

[14] Shelton, E. G. H. et Cho, A. Y., J. Appl. Phys. 37 (1966) 3544.

[15] Modak, A. T. et Pagni, P. J., J. Chem. Phys. 59 (1973) 2019.

[16] Cho, A. Y. et Hendricks, C. D., J. Appl. Phys. 40 (1969) 3339.

[17] WACH, J., Colloque International, CNRS no 205, Odeillo (1971) 293. 